\title{
Mineralocorticoid Secretion Process
}

National Cancer Institute

\section{Source}

National Cancer Institute. Mineralocorticoid Secretion Process. NCI Thesaurus. Code C41503.

Mineralocorticoid Secretion Induction consists of initiation of activities involved in release into the bloodstream of a class of C21 corticosteroids (mineralocorticoids) involved in regulation of electrolyte homeostasis (sodium retention and potassium loss) and water balance (retention) through effects on epithelial ion transport. Alteration of positive or negative feedback mechanisms can affect hormone release. 